\title{
An investigation of the perceptual basis of redundancy gain and orthogonal interference for integral dimensions
}

\author{
JAMES R. DYKES, JR. \\ University of Texas at San Antonio, San Antonio, Texas 78285 \\ and \\ ROBERT G. COOPER, JR. \\ University of Texas at Austin, Austin, Texas 78712
}

\begin{abstract}
Redundancy gain and orthogonal interference for height and width were demonstrated in two experiments using a relative coding task with number of intertrial repetitions controlled. Orthogonal interference was shown to be perceptually based rather than simply an intertrial repetitions effect as suggested by Felfoldy (1974). These results from a relative coding task were discussed in terms of the previous multidimensional processing literature. It was concluded that Lockhead's (1972) model has been applied too generally. An alternative model (a parallel, dimensional analysis stage followed by a stage in which dimensional information is integrated) was suggested.
\end{abstract}

The effect of processing information from stimuli which vary in more than one dimension has recently received substantial theoretical and empirical attention. Garner and Felfoldy (1970) have shown that certain pairs of dimensions produce redundancy gain and orthogonal interference in a speeded classification task. Reaction time is shorter for stimuli which differed along both dimensions in a perfectly correlated fashion than for stimuli differing along either individual dimension when the other dimension was held constant. This phenomenon is known as redundancy gain. On the other hand, reaction time is longer for classifying stimuli along one dimension when the second dimension is orthogonally varied than when the second dimension is held constant. This phenomenon is known as orthogonal interference. Consistent with Lockhead's (1966) terminology, Garner (1970) called pairs of dimensions which showed both redundancy gain and orthogonal interference integral and further suggested that: "Two dimensions are integral if in order for a level on one dimension to be realized, there must be a dimensional level specified for the other" (p. 354). Several models have been proposed to account for the integrality effects.

Garner (1970) has suggested that subjects can redefine stimuli varying along two integral dimensions into a single, new dimension. Stimuli differing along both stimulus dimensions in a correlated fashion should be more discriminable in the new dimension than stimuli differing along only one stimulus dimension. For instance, rectangles differing in both height and width should be more dis- criminable in terms of surface area or shape than rectangles differing in only height or only width. Hence classification should be faster and redundancy gain should be evidenced. Conversely, having to selectively attend to one integral dimension could be expected to produce interference for any of a variety of reasons (e.g., an additional stage of processing, misallocation of attention, response interference).

Felfoldy (1974) suggested one possible source of interference. In his experiment, Felfoldy examined the effect of intertrial repetitions on redundancy gain and orthogonal interference in an absolute classification task. He found that orthogonal interference showed an intertrial repetition effect, while redundancy gain did not. Felfoldy concluded that the greater number of alternatives in the comparison process and the consequently lower frequency of stimulus repetitions in orthogonal interference conditions caused the reaction time difference between orthogonal interference conditions and the single dimension conditions. Since reaction time is longer for nonrepetition trials than for repetition trials, average reaction times should be longer in the orthogonal interference conditions than in the single dimension conditions.

Felfoldy's suggestion should be tested with a paradigm which is basic enough to insure that the subject's earliest stages of processing are being measured, but which has enough different stimuli to control the number of stimulus repetition trials across the redundancy gain, single dimension, and orthogonal interference conditions. The data previously discussed have been generated from ab- 
solute coding tasks; the subject identifies each stimulus in order to determine its appropriate classification. Alternatively, if a subject is told to pick the taller of two rectangles which are simultaneously presented, a relative judgment is required. On one trial, a particular rectangle may be the taller and on the next trial the shorter. Such a relative coding task meets both constraints of the criterion. With highly discriminable rectangles, the task is simple and the subject only has to choose between two responses. Simultaneously, the number of stimuli can be increased such that no stimulus repetition trials occur in any condition.

If the paradigm is effective, redundancy gain should be evidenced in the relative coding task. However, if orthogonal interference is simply an artifact of the number of stimulus repetition trials, then no orthogonal interference should be evidenced in the relative coding task. Alternatively, if varying an irrelevant dimension is a source of perceptual or response interference in its own right, then both redundancy gain and orthogonal interference should be evidenced.

Using a relative coding task, redundancy gain was tested in Experiment 1 and orthogonal interference in Experiment 2. Rectangles varying in height and width were used as stimuli. The height and width dimensions meet Garner's (1970) definition of integrality, and Felfoldy (1974) has shown that they exhibit redundancy gain (although this was not significant in his first experiment) and orthogonal interference in an absolute coding task. In each experiment here, the effect was tested across three levels of discriminability of each dimension.

\section{EXPERIMENT 1}

\section{Method}

Subjects. One female and seven males (including the authors) served as subjects. All had normal vision.

Stimuli and Apparatus. The stimuli were slides of pairs of black rectangles differing in height, width, or both. One rectangle was centered in a white square on the left and the other was centered in a square on the right. The white square on the left was positioned $3.5 \mathrm{~cm}$ higher than the one on the right so that height differences could not be determined simply by a horizontal scan. The rectangles within a pair could differ in four ways: height only, width only, height and width positively correlated (one rectangle taller and wider than the other), and height and width negatively correlated (one rectangle taller and narrower than the other). A set of slides was generated for each of these four differences. For each set, the rectangles within a pair differed by one of three magnitudes ( 5,7 , and 9 JNDs) according to Ono's equation (Ono, 1967). In each slide, one of the rectangles was always $3.7 \mathrm{~cm}$ wide and $9.7 \mathrm{~cm}$ high. For each magnitude in each set, the $3.7 \times 9.7 \mathrm{~cm}$ rectangle was paired with two variable rectangles: one larger on the relevant dimension(s) and one smaller on the variable dimension(s). Table 1 shows the dimensions of these variable rectangles. Thus, within each set, there were two pairs of rectangles for each of the three JNDs. For each of those exemplars there were 6 slides ( 3 for each of the left/right positions). Thus, there were $36(3 \times 2 \times 2 \times 3)$ slides within each set.
A Kodak Carousel projector presented the stimuli on a screen suspended $84.5 \mathrm{~cm}$ in front of the subject. Thus the $3.7 \times 9.7 \mathrm{~cm}$ rectangle subtended $2^{\circ} 30^{\prime} \times 6^{\circ} 34^{\prime}$ of visual angle. A white light contrally located on the screen was illuminated for the $3 / 4 \mathrm{sec}$ preceding each stimulus slide. This served as a fixation point and a warning signal. Reaction time from stimulus onset was measured with an Industriai Timer llock. The lock was stopped by a handheld response box with two buttons (one on the left and one on the right).

Design and Procedure. Each of the four stimulus sets was run in a block of 36 test trials with JND conditions randomized within each block ( 12 trials per block). Four orders of blocks were used according to a 4 by 4 digram-balanced Latin square (Wagenaar, 1969). For each block, there were two target conditions: Target 1 (the larget was the taller, the wider, the taller and wider, or the taller and narrower) and Target 2 (the target that the shorter, the narrower, the shorter and narrower, or the shoster and wider). Two subjects served in each of the four orders of blocks. One of these had the Target 1 condition on Day, and Target 2 on Day, while the other subject had the Target 2 condition on Day, and Target 1 on Day - Target 1 and Target 2 were run on differest days in order 10 minimize interference effects.

Each subject was instructed to push the bution on the same side as the target as quickly as possible and was informed that both his choice and time would be recorded. Before each block, the subject was informed as to how the rectangles in each pair would differ and what the correct choice would be for that block (e.g., "In each pair, one rectangle will be taller and wider than the other. You should pick that rectangle."). Next, the subject was shown 10 practice slides randomly chosen within the appropriate 36-slide set and then was shown the entire 36-slide set as test trials. At the end of the four blocks on Day, , the subject was informed that Day ${ }_{2}$ would be the same except that the opposite choice would be the target for each block.

\section{Results}

The median RT for each subject for each JND within each set within each target condition was computed. A four-way analysis of variance (Order of Blocks by Stimulus Sets by JND by Targets) was performed on the median RT per subject. The Order and the Targets effects were not significant ( $F s<1.0$ ). The Sets effect approached significance $[F(3,12)=$ $2.65 ; .05<p<.10]$. Essentially all of the Sets effect variance was due to $R T$ being significantly longer for the height and width conditions than for the positively and negatively correlated conditions $[\mathrm{F}(1,12)=$ $7.74, \mathrm{p}<.025]$. There was no difference between height and width or between positively and negatively

Table 1

Projected Dimensions of the Variable Rectangles for Each Stimulus Set in Experiment 1

\begin{tabular}{rrrrrr}
\hline & \multicolumn{5}{c}{ Stimulus Set } \\
\cline { 2 - 6 } JND & Height & Width & $\begin{array}{l}\text { Positively } \\
\text { Correlated }\end{array}$ & $\begin{array}{l}\text { Negatively } \\
\text { Correlated }\end{array}$ \\
\hline \multirow{2}{*}{5} & $3.7 \times 8.4$ & $3.1 \times 9.7$ & $3.1 \times 8.4$ & $4.3 \times 8.4$ \\
& $3.7 \times 11.2$ & $4.3 \times 9.7$ & $4.3 \times 11.2$ & $3.1 \times 11.2$ \\
7 & $3.7 \times 8.0$ & $3.0 \times 9.7$ & $3.0 \times 8.0$ & $4.6 \times 8.0$ \\
& $3.7 \times 11.8$ & $4.6 \times 9.7$ & $4.6 \times 11.8$ & $3.0 \times 11.8$ \\
9 & $3.7 \times 7.6$ & $2.9 \times 9.7$ & $2.9 \times 7.6$ & $4.8 \times 7.6$ \\
& $3.7 \times 12.4$ & $4.8 \times 9.7$ & $4.8 \times 12.4$ & $2.9 \times 12.4$ \\
\hline
\end{tabular}

Note-All dimensions in centimeters. The other rectangle in each pair was always $3.7 \mathrm{~cm}$ wide $\times 9.7 \mathrm{~cm}$ high. 
correlated conditions (Fs $<1.0$ ). RT decreased as number of JNDs increased $[F(2,8)=12.38$, $\mathrm{p}<.01\}$. The decrease in RT across JNDs was linear $[F(1,8)=24.46, p<.001]$. Only one interaction was significant: the Stimulus Sets by Order of Blocks by JND interaction $[F(18,24)=2.18, p<.05]$. In our Latin square design, Order of Blocks was the row variable, Stimulus Sets was the column variable, and Blocks was the Latinized variable. To see if the three-way interaction was due to a Blocks by JND interaction, a Latin square analysis (Winer, 1962) was performed. The Blocks by JND interaction was significant $[F(6,24)=3.00, p<.05]$ and the Latin square residual was not $[\mathrm{F}(12,24)=1.78, \mathrm{p}>.10]$. The interaction was predominantly due to the linear JND effect being significantly larger for each subject's first block of trials than for subsequent blocks on each day $[F(1,24)=13.33, p<.01]$. The mean RTs and percent errors for each JND in each stimulus set are shown in Table 2 .

In order to be certain that the differences in RT for Sets and JND were not due to a speed-accuracy tradeoff, a two-way analysis of variance (Sets by JND) was performed on the mean number of errors. The differences between sets were not significant $(F<1)$. There was a significant JND effect $[F(2,14)$ $=27.94, \mathrm{p}<.001]$. There was not a significant difference between 7 and 9 JNDs $(F=1.26)$, but more errors were made for the 5 JND stimuli than for the 7 JND stimuli $[F(1,14)=45.42, p<.001]$. The Set by JND interaction was not significant $(\mathrm{F}<1)$.

To summarize, redundancy gain was demonstrated for height and width of rectangles in a relative coding task with no stimulus repetitions. The JND effect was significant, but did not interact with redundancy gain. The only difference in error rate was positively related to RT; increasing the number of JNDs decreased both RT and error rate. Thus, neither the redundancy gain nor the JND effect can be explained in terms of a speed-accuracy tradeoff. Thus, the sensitivity of the relative coding task was demonstrated.

To test Felfoldy's (1974) suggestion, it was necessary to investigate orthogonal interference for height and width in a relative coding task in which the number of stimulus repetitions was controlled. This was done in Experiment 2.

\section{EXPERIMENT 2}

\section{Method}

In describing Experiment 2, only the differences from Experiment 1 will be discussed.

Subjects. The eight subjects (six females and two males) were volunteers from the Perception and Attention courses at the University of Texas at San Antonio.

Stimuli and Apparatus. Four new sets of slides were made from the slides used in Experiment 1. The new height-only set was composed of 24 slides from the height set (8 for each JND). Similarly, the new width-only set was composed of 24 slides from the width set ( 8 for each JND). Two slides for each JND were taken from the height, positively correlated, and negatively correlated sets from Experiment 1. These slides comprised a set (height orthogonal) in which each pair of rectangles differed in height, but, for each magnitude of height difference, width varied orthogonaily. Similarly a set of slides (width orthogonal) was constructed such that the rectangles differed in width while height was orthogonally varied. The slides were rear-projected on a frosted acetate screen and RT was measured to the nearest millisecond by a Hunter Klockounter.

Design and Procedure. In the height-only condition, the subjects were told that width would be constant and that they were to pick the taller rectangle on one day and the shorter on the other day. In the width-only condition, the subjects were told that height would be constant and that they were to pick the wider on one day and the narrower on the other day. In the height orthogonal condition, the subjects were told that variations in width should be ignored and that they were to pick the taller on one day and the shorter on the other day. In the width orthogonal condition, the subjects were told that variations in height should be ignored and that they were to pick the wider on one day and the narrower on the other day.

There were 36 test trials for each condition. For height only and width only, the 36 test trials were presented by the 24 slides in forward order and the last 12 in reverse order. For height orthogonal and width orthogonal, the 36 test trials were presented by the 18 slides in forward and then reverse order. Except for the reversal, the same stimulus was not shown on consecutive trials in any block.

The counterbalancing of target (taller/shorter and wider/ narrower) across days and practice was the same as in Experiment 1.

\section{Results}

The median RT for each subject for each JND within each set within each target condition was

Table 2

Mean Reaction Time (Milliseconds) and Mean Proportion of Errors for Experiment 1 for Each Stimulus Set

\begin{tabular}{ccccccc}
\hline & & \multicolumn{5}{c}{ Set } \\
\cline { 2 - 6 } JND & Height & Width & $\begin{array}{c}\text { Positively } \\
\text { Correlated }\end{array}$ & $\begin{array}{c}\text { Negatively } \\
\text { Correlated }\end{array}$ & Mean \\
\hline 5 & $482.19(.09)$ & $485.63(.15)$ & $470.31(.09)$ & $470.63(.09)$ & $477.19(.11)$ \\
9 & $471.56(.03)$ & $485.00(.04)$ & $458.13(.05)$ & $459.06(.03)$ & $468.44(.04)$ & $455.55(.03)$ \\
Mean & $470.00(.04)$ & $465.94(.01)$ & $446.25(.01)$ & $440.00(.04)$ & $456.56(.06)$ & $467.06(.06)$ \\
\hline
\end{tabular}


computed. A four-way analysis of variance (Order of Blocks by Stimulus Sets by JND by Targets) was performed on the median RT per subject. As in Experiment 1 , the Order and the Targets effects were not significant $(\mathrm{Fs}<1.0)$. The Sets effect was significant $[F(3,12)=8.66, p<.01]$. This was due to orthogonal interference. RT was longer for height orthogonal and width orthogonal than for height only and width only $[F(1,12)=19.54, p<.01]$. The other components of variance in the Sets effect (height sets being slightly faster than width sets and the orthogonal interference being slightly greater for width than for height) were sizable, but not significant $[\mathrm{Fs}(1,12)=3.70$ and $2.75, \mathrm{p}>.05$ and $\mathrm{p}>.10$, respectively]. RT decreased as JND increased $[F(2,8)=24.05, p<.01]$. The only significant interaction was Sets by JND $[F(6,24)=6.83, p<.01]$. This is apparently due to a floor effect; the JND effect was smaller for sets with short average RTs than for sets with long RTs. Height only and width only were faster than height orthogonal and width orthogonal and the JND effect was smaller $[F(2,24)$ $=14.29, \mathrm{p}<.01]$. The height sets were nonsignificantly faster than the width sets, and the JND effect was smaller $[F(2,24)=25.11, p<.01]$. The mean RTs and percent errors for each JND in each stimulus set are shown in Table 3.

In order to determine if a speed-accuracy tradeoff was responsible for the RT differences, a two-way analysis of variance (Set by JND) was performed on the mean number of errors. The differences between sets were not significant $[\mathrm{F}(3,12)=1.49$; $.10<p<.25]$. The JND effect approached significance $[F(2,14)=3.14 ; .05<p<.10]$ and the significant linear trend across $\operatorname{JNDs}[F(1,14)=6.70$, $\mathrm{p}<.025]$ indicated that error rate decreased as number of JNDs increased. The Set by JND interaction was not significant $(F<1.0)$.

To summarize, orthogonal interference was demonstrated for height and width of rectangles in a relative coding task with number of stimulus repetitions controlled. RT decreased as JNDs increased, but this effect was smaller for sets with short average RTs than for sets with long RTs, The only difference in error rate was positively correlated with RT; increasing the number of JNDs decreased both RT and error rate. Thus there was no evidence of a speed-accuracy tradeoff, and the RT effects can be interpreted independently of the error rate.

\section{DISCUSSION}

The most important evidence from these experiments was the clear demonstration of redundancy gain and orthogonal interference in a relative coding task. These results will be discussed with respect to two issues. First, they will be used to refute Felfoldy's (1974) explanation of orthogonal interference. Then they will be used in concert with the results from several other studies to support a modification of Lockhead's model of multidimensional information processing.

The present study replicated Felfoldy's (1974) findings that the height and width dimensions of rectangles exhibit both redundancy gain and orthogonal interference, but our results show that Felfoldy's explanation of orthogonal interference is at least incomplete. In our paradigm, the subject must encode relative information about the stimulus rectangles; absolute information will not be helpful. For instance, in each display one of the rectangles was $9.7 \mathrm{~cm}$ high and $3.7 \mathrm{~cm}$ wide. On half the trials this was the correct choice, and on the other half it was the incorrect choice. Thus there is no memory matching stage in the processing of our stimuli. Further, the lack of repetitions of stimuli in our experiment precluded any repetition effect. Thus it can be concluded that orthogonal interference as well as redundancy gain arises as a function of the stimulus encoding process.

These data also contribute to a rich literature and should be considered within that context for hypothesizing a unified model of multidimensional processing. First, these data are compatible with Garner's (1970) model, as previously described. If that model is correct, redundancy gain would be the result of increased discriminability within a single "new" dimension, such as surface area or shape. The source of orthogonal interference would be unspecified, except that a simple stimulus repetitions effect has been eliminated as a complete explanation.

Alternatively, both these data and Garner's data

Table 3

Mean Reaction Time (Milliseconds) and Mean Proportion of Errors for Experiment 2 for Each Stimulus Set

\begin{tabular}{ccccccc}
\hline & \multicolumn{5}{c}{ Set } & \multicolumn{2}{c}{ Width } \\
\cline { 2 - 6 } JND & Height Only & Width Only & Height & Orthogonal & Orthogonal & Mean \\
\hline 5 & $506.47(.03)$ & $525.34(.01)$ & $545.50(.03)$ & $607.53(.02)$ & $546.21(.02)$ & $525.87(.01)$ \\
Mean & $503.28(.01)$ & $495.47(.01)$ & $542.75(.01)$ & $561.97(.01)$ & $514.43(.01)$ & $528.84(.01)$ \\
\hline
\end{tabular}

Note-Proportion of errors given in parentheses. 
are compatible with a model in which the two stimulus dimensions are processed by separate analyzers operating in parallel. Biederman and Checkosky (1970) propounded a "fastest horse model" to account for redundancy gain. Assuming that the processing time required by each dimensional analyzer is a continuous variable with nonzero variance, different analyzers would finish first on different trials. With redundant information available in both dimensions, subjects should be able to initiate the response as soon as the faster analyzer was finished on each trial. Consequently, reaction time should be faster on the average in the redundant condition than in single dimension conditions. Orthogonal interference could either be due to a misallocation of attention between the parallel analyzers or due to response interference (Egeth, 1967).

This model, however, has recently been shown to be inadequate in accounting for data generated in more difficult and complex tasks. Part of the data comes from investigating two different redundancy gain conditions: a linearly correlated condition (represented by Xs in Figure 1) and a sawtooth condition (represented by Os in Figure 1). In both conditions, each level of one dimension is paired with one and only one level of the other dimension. If dimensional information were maintained separately throughout an identification task, then performance should be the same for the linearly correlated condition as for the sawtooth condition. The dimensional information is identical in the two conditions; only the relationship between levels of the two stimulus dimensions is different. However, Lockhead (e.g., Lockhead, 1970; Monahan \& Lockhead, 1977) has shown that accuracy is higher and reaction time is shorter in the sawtooth condition than in the linearly correlated condition. To account for these data, the information from the two stimulus dimensions must be integrated at some stage before the response is made.

Additional evidence that dimensional information is integrated prior to the response has been reported by Weintraub (1971). He compared identification performance between different subsets of 15 rectangles. In terms of decreasing identification accuracy, the subsets were ordered as follows: sawtooth, negatively correlated (the tall rectangles were narrow), height or width alone, and positively correlated (squares, the tall rectangles were wide). Weintraub suggested that height and width information is integrated to allow a judgment based on shape. This explanation adequately accounts for the reported ordering of subsets, including the finding that the positively correlated set led to the poorest performance. Although integration of dimensional information was demonstrated, it is important to

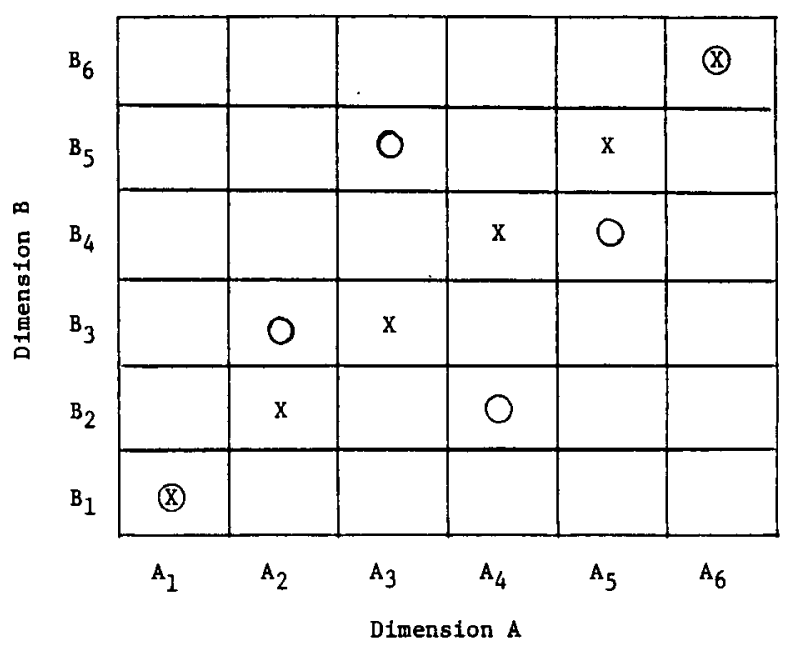

Figure 1. Stimulus pairing used by Monahan and Lockhead (1977). The Xs represent the linearly correlated set and the Os represent the sawtooth set.

note that in Experiment 1 we found redundancy gain for the positively correlated stimuli.

Attempting to account for both his data and Garner's data, Lockhead (1972) proposed that the processing of integral multidimensional stimuli is accomplished by locating them in an n-dimensional, Euclidean, psychological space. Such a model elegantly predicts Lockhead's data. As can be seen in Figure 1, the sawtooth stimuli would be further apart than the linearly correlated stimuli in a psychological space with axes corresponding to height and width. The model can also account for Weintraub's data if one assumes that at least one of the n-dimensions of psychological space is based on some sort of integrated "shape" information. Monahan and Lockhead (1977) present substantial data that this is the case and further propose prime candidates for what the "shape" dimensions might be.

In order to account for Garner and Felfoldy's (1970) data, Lockhead suggested that the processing of stimuli into psychological space is the first stage of processing that subjects are able to use in order to make a response. In other words, stimuli are initially holistically processed, and, in a later stage of processing, dimensional information becomes available. Such a model can explain Garner and Felfoldy's results in isolation. Stimuli differing along two stimulus dimensions should be more distant from each other in psychological space (and more discriminable) than stimuli differing along a single stimulus dimension. This would generate the redundancy gain. Requiring the subject to selectively attend to one dimension would necessitate a second, dimensional stage of processing in his model. Thus orthogonal interference would be the result of additional analysis. 
However, this ordering of the two stages cannot account for our data. The difference between the absolute and relative tasks is that the absolute task can be solved by locating the stimulus in psychological space (the first stage of processing), but the relative task can only be solved by analyzing the stimuli into dimensions (the second stage of processing). Thus, there would be no reason to predict either redundancy gain or orthogonal interference in a relative coding task. Since there is no mechanism proposed by which knowledge of location in psychological space leads to faster processing of dimensional information, there is no reason to predict redundancy gain. Similarly, since all of the judgments are made in the same second stage, there is no mechanism to explain why reaction time should be longer in the orthogonal interference conditions than in the single dimension conditions. It is important to note that our task employs pairs of integral stimuli. It is possible to argue that Lockhead's model cannot be applied to our task. However, our task produces redundancy gain and orthogonal interference similar to that in Garner and Felfoldy's (1970) task. These are phenomena that Lockhead has tried to explain with his model, and we will propose an alternative procedure for integrating these new data.

All of the reviewed data can be explained by a model in which the order of the stages in Lockhead's model is reversed: a dimensional analysis stage followed by a stage in which the dimensional information is integrated to give a location in an ndimensional psychological space. The experiments whose results can be explained by the first stage of processing (e.g., Felfoldy, 1974; Garner, 1970; and our results) share several characteristics in common: the response is a binary choice, reaction times are on the order of $1 / 2 \mathrm{sec}$, and redundancy gain is exhibited for both positively and negatively correlated stimulus sets. For these results, redundancy gain would be explained by Biederman and Checkosky's (1970) "fastest horse" model and orthogonal interference would be explained either by misallocation of attention to the irrelevant analyzer or by response interference.

The experiments which require an integration of dimensional information to explain their results (e.g., Lockhead, 1972; Weintraub, 1971) exhibit a different set of characteristics, some of which indicate a more complicated and later stage of processing. Three characteristics suggest that more complicated processing occurs for these tasks. First, these tasks have increased memory load deriving from the larger response sets $(6,7,10$, or 15 responses). Second, reaction times are longer, on the order of $1 \mathrm{sec}$. Third, the superiority of performance in the sawtooth condition over the positively correlated condition appears to require extensive prac- tice, while redundancy gain and orthogonal interference are exhibited by naive subjects after only limited practice (e.g., 10 practice trials in our study). In an experiment with four response alternatives, Levy and Haggbloom (1971) found that reaction time was faster for the sawtooth condition only for the last 240 trials, and faster for the linearly correlated condition during the first 240 trials. This last result and one other characteristic suggest that a different kind of processing is responsible for the results of these experiments. Redundancy gain is only exhibited for the negatively correlated stimulus set. Comparing the positively correlated set to the single dimension sets, Weintraub (1971) reported less information transmitted and Monahan and Lockhead (1977) reported longer reaction times and more errors. We propose that the different kind of processing that produces these results and that distinguishes these studies from the first group is exactly the stage proposed by Lockhead, locating the stimulus in an $\mathrm{n}$-dimensional psychological space and responding on the basis of that location.

In summary, the data from our two experiments provide two forms of negative evidence for other models. They show that Felfoldy's (1974) model is an inadequate model of orthogonal interference, and they demonstrate redundancy gain and orthogonal interference in a situation for which Lockhead's model (1972) cannot account. This latter result was used in conjunction with a task analysis and an analysis of discrepant results from other studies of multidimensional information processing to support a modification of Lockhead's model. The particular alternative model proposed, dimensional processing followed by integration of dimensional information, is one of many alternatives. The central point was to show that Lockhead's demonstration of the inadequacy of a "fastest horse" model and his account of redundancy gain had been applied too generally, and ought to be limited to a smaller class of phenomena.

\section{REFERENCES}

Biederman, I., \& Checkosky, S. F. Processing redundant information. Joumal of Experimental Psychology, 1970, 83, 486-490.

Egetr, H. Selective attention. Psychological Bulletin, 1967, 67, 41-57.

FeLfoldy, G. L. Repetition effects in choice reaction time to multidimensional stimuli. Perception \& Psychophysics, 1974, 15, 453-459.

Garner, W. R. The stimulus in information processing. American Psychologist, 1970, 25, 350-358.

GARNER, W. R., \& FELFOLDY, G. Integrality and separability of stimulus dimensions in information processing. Cognitive Psychology, 1970, 1, 225-241.

LEVY, R. M., \& HagGbloom, S. J. Test of a multidimensional discrimination model of stimulus identification. Psychonomic Science, 1971, 25, 203-204. 
LOCKHEAD, G. R. Effects of dimensional redundancy on visual discrimination. Joumal of Experimental Psychology, 1966, 72, 95-104.

LOCKHEAD, G. R. Identification and the form of multidimensional discrimination space. Journal of Experimental Psychology, 1970, 85, 1-10.

Lockhead, G. R. Processing dimensional stimuli. Psychological Review, 1972, 79, 410-419.

Monahan, J. S., \& LOCKHEAD, G. R. Identification of integral stimuli. Journal of Experimental Psychology: General, 1977, 106, 94-110.

ONo, H. Difference threshold for stimulus length under simulta- neous and nonsimultaneous viewing conditions. Perception \& Psychophysics, 1967, 2, 201-207.

WAGENAAR, W. A. Note on the construction of digram-balanced Latin squares. Psychological Bulletin, 1969, 72, 384-386.

Weintraub, D. J. Rectangle discriminability: Perceptual relativity and the law of prägnanz. Journal of Experimental Psychology, $1971,88,1-11$.

WINER, B. I. Statistical principles in experimental design. New York: McGraw-Hill, 1962.

(Received for publication July 6, 1977; revision accepted October 20,1977.) 